\title{
Identifying an optimal protocol for resting metabolic rate measurement in recreationally active university students
}

\author{
T. Acheson ${ }^{1}$, A. Dooley ${ }^{1}$, F. McGirr ${ }^{1}$, S. Kilcullen ${ }^{1}$, S. McGrath ${ }^{1}$, A. Ezenwa ${ }^{1}$, K. Horner $^{1}$ and \\ D. Crognale ${ }^{2}$ \\ ${ }^{1}$ School of Public Health, Physiotherapy and Sports Science, University College Dublin and \\ ${ }^{2}$ Institute of Sport and Health, University College Dublin, Ireland
}

Resting metabolic rate (RMR) is the energy required and utilised by the body, in a rested state. During RMR measurement, current best practice recommendations are to discard the first 5 minutes of data, and then use a validated definition for RMR steady state (SS) such as 5-min of $\mathrm{SS}^{(1)}$. However, a wide variety of test durations and protocols are often used by researchers and practitioners, with some studies assessing mean RMR over a fixed duration. Knowledge of the accuracy of different protocols is important when interpreting findings and assessing the optimal protocol to use in a population. This study investigated (1) the accuracy and (2) the reproducibility of 6 different RMR measurement protocols against the widely accepted 5-minute SS protocol when measured in recreationally active university students.

This study was a cross-sectional study and took place in a physiology laboratory. A total of 19 (11 male, 8 female) recreationally active college students (age $=20.58 \pm 0.69$ years, height $=174.15 \pm 9.08 \mathrm{~cm}$, weight $=69.45 \pm 11.74 \mathrm{~kg}$ ) completed a 30 -minute RMR test and 12 participants completed a second test one week after the first.

Participants had to be recreationally active ( $>3$ days/week), a university student and without any underlying conditions which may impact RMR measurement.

RMR was calculated using indirect calorimetry through use of a metabolic cart (CosMed Quark CPET (Cosmed Srl, Rome, Italy)) and ventilated hood. We compared 10-minute SS and 10,15,20,25,30-minute fixed time (FT) to the 5-minute SS 'best practice' method. These protocols were statistically analysed through the use of Intra-Class Correlation Coefficients (ICC), Paired T-Tests and Bland-Altman plots to determine differences in the resulting RMR. Coefficients of variation of the two repeated test days were also calculated.

Out of the 7 analysis methods used, RMR values of $1967.67 \pm 317.33$ (10-minute SS) and $1970.64 \pm 315.9$ (30-minute FT) were similar to the 5-minute SS (1970.22 \pm 333.41$)$. However, the four shorter FT duration protocols differed significantly to 5-minute SS ( $\mathrm{p}<0.05)$. The ICCs comparing Visit 1 versus Visit 2 showed excellent correlation $(0.91-0.93)$, regardless of what protocol was used. In addition, the reproducibility of all test protocols was excellent producing mean CVs of $<5 \%$.

In recreationally active university students, only 10-minute SS and 30-minute FT protocols give as accurate results as the 5-minute SS 'best practice' protocol. The shorter FT protocols were not as accurate and should be avoided. The excellent reproducibility found with all protocols shows that two sessions for RMR testing may not be necessary, making testing more accessible. This has a significant practical application as the need for only one test allows the process to be more time-efficient and accessible for larger cohorts such as teams or research studies looking at large populations.

\section{References}

1. Fullmer et al. (2015) J Am Diet Assoc. 2015; 115(9):1417-1446. 